\title{
Effect of Collagen-Enhanced Fibrin Sealant on Seroma Formation in a Rat Mastectomy Model
}

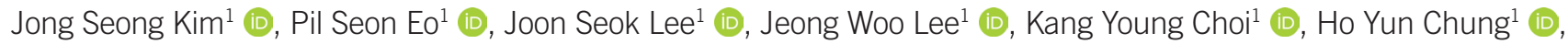 \\ Byung Chae Cho $^{1}$ (D), Jeeyeon Lee ${ }^{2}$ (D), Ho Yong Park² (D), Jung Dug Yang ${ }^{1}$ (D) \\ Departments of ${ }^{1}$ Plastic and Reconstructive Surgery and ${ }^{2}$ Surgery, School of Medicine, Kyungpook National University, Daegu, Korea
}

\begin{abstract}
Background: Seromas are caused by leakage of lymphovascular fluid into postoperative dead space. This is the most common complication after reconstructive breast surgery. The purpose of this study is to demonstrate the utility of seroma-preventing substances by using a collagen-enhanced fibrin sealant on a rat mastectomy model.

Methods: Thirty-six Sprague-Dawley rats were divided into three groups. After mastectomy and axillary lymph node dissection, normal saline was applied to the dead spaces in group 1 (control). In group 2, a collagen-enhanced fibrin sealant was applied, and in group 3, triamcinolone acetate solution was applied. Afterwards, the amount of seroma was measured by three-dimensional micro-computed tomography (3D micro-CT) volumetry analysis and manual aspiration after 7 and 14 days, respectively.

Results: The volume of seroma were significantly reduced in groups 2 and 3 compared to group 1 in both 3D micro-CT volumetry analysis and manual aspiration on postoperative day $7(\mathrm{P}<0.001)$. In addition, the results observed in day 14 also showed a decrease in the amount of seroma analyzed by CT in groups 2 and 3 compared to group $1(P<0.05)$. In histopathologic examination inflammation was observed more frequently in group 1 and angiogenesis was more active in group 2.

Conclusion: The use of a collagen-enhanced fibrin sealant (Collaseal) is as effective as triamcinolone control injected in a rat mastectomy model.
\end{abstract}

Keywords: Fibrin tissue adhesive; Seroma; Mastectomy

\section{Introduction}

In reconstructive breast surgery, the formation of seroma is difficult to prevent. Various risk factors such as smoking, obesity, radiotherapy, and chemotherapy have been reported as the cause of seroma. Consensus on the best way to prevent or treat seroma has not yet been established [1,2]. There are currently various methods to reduce seroma in practice, such as the quilting suture method, compression therapy, and sclerosing agents. Sclerosing agents include fibrin glue, tetracycline, hydrochloric acid, triamcinolone, talc, and Abnoba Viscum. Though these chemical injections have been noted as an alternative to surgery for treating refractory seroma, research on wound healing and seroma prevention using such materials in the form of patches during surgery has been inadequate [3-5].

Collaseal collagen-enhanced fibrin sealant is a form of membrane containing type 1 collagen, hyaluronic acid, thrombin and levodopa. It absorbs exudates, helps heal wounds, enhances adhesion to tissues and helps wound hemostasis. The major component, collagen, improves the adherence, proliferation and migration of fibroblasts and has chemotactic activity on cells involved in healing, such as granulocytes and macrophages. It also forms new tissue by acting as a bridge between the edges of damaged tis-

\section{Original Article}

Received: August 21, 2020

Revised: October 9, 2020

Accepted: October 9, 2020

Corresponding author:

Jung Dug Yang, M.D., Ph.D.

Department of Plastic and Reconstructive Surgery, School of Medicine, Kyungpook National University, 130 Dongdeok-ro, Junggu, Daegu 41944, Korea

Tel: +82-53-420-5685

Fax: +82-53-425-3879

E-mail: lambyang@knu.ac.kr

This is an Open Access article distributed under the terms of the Creative Commons Attribution Non-Commercial License (https://creativecommons.org/licenses/by-nc/4.0/) which permits unrestricted non-commercial use, distribution, and reproduction in any medium, provided the original work is properly cited.

C) 2020 Korean Wound Management Society 
sue. It thus helps wound recovery by providing the ideal components needed in the recovery phase of damaged tissue [6-8].

In this study, seroma models with Sprague-Dawley (SD) rats were prepared to evaluate the effect on seroma prevention of a collagen-enhanced fibrin sealant (Collaseal) compared to triamcinolone and saline through histological examination and three-dimensional micro-computed tomography (3D microCT) parameters [9-11].

\section{Methods}

The approval of the study was obtained from the Local Ethical Committee of the Experimental Animal Laboratory at Daegu Gyeongbuk Medical Innovation Foundation (approval No. DGMIF D18017). Thirty-six male SD rats (average weight, 200-300 g) were used in the experiment. The rats were divided into three groups of 12 rats each. All rats were fed amounts of feed sufficient for SD rats. The feeding room was climate controlled with a temperature of $23^{\circ} \mathrm{C} \pm 3^{\circ} \mathrm{C}, 50 \% \pm 20 \%$ humidity, 10-20 times/hr. ventilation, and an illuminance of 150-300 lux with 12-hour day/night cycles.

All SD rats received one-sided (right) mastectomy and axil- lary lymph node dissection as described by Harada et al. [9]. Prophylactic antibiotics were administered by intramuscular injection of gentamicin, while meloxicam was subcutaneously injected as an analgesic agent. Six milligrams of Zolazepam (Zoletil; Virbac, Seoul, Korea) and 1.5 mg of Xylazine (Rompun; Bayer, Leverkusen, Germany) were injected intraperitoneally as general anesthetics. The anterior thoracic and right axilla area were shaved and sterilized with $10 \%$ povidone solution.

A $2 \mathrm{~cm}$-vertical incision was made from the jugular notch to the xiphoid, elevating the subcutaneous flap. After removing the pectoralis major muscle and the axillary lymph node, the axillary artery, vein, and nerve were preserved. After the dissection, $0.4 \mathrm{~mL}$ of saline was administered to the control group (group 1). In the experimental group (group 2), Collaseal (Dalim Tissen Co., Ltd., Seoul, Korea) was trimmed into a membrane of $1 \mathrm{~cm}^{2}$ and applied to the side of lymph node dissection. In the other experimental group (group 3), about $1 / 300$ of a normal human dose of triamcinolone was measured, and was included in $0.4 \mathrm{~mL}$ of mixed solution $(0.382$ $\mathrm{mL}$ of saline with $0.018 \mathrm{~mL}$ of triamcinolone) that was administered to each rat. Afterwards, wound closure was done in all groups using Ethilon 5-0 skin sutures (Fig. 1). Povidone-io-
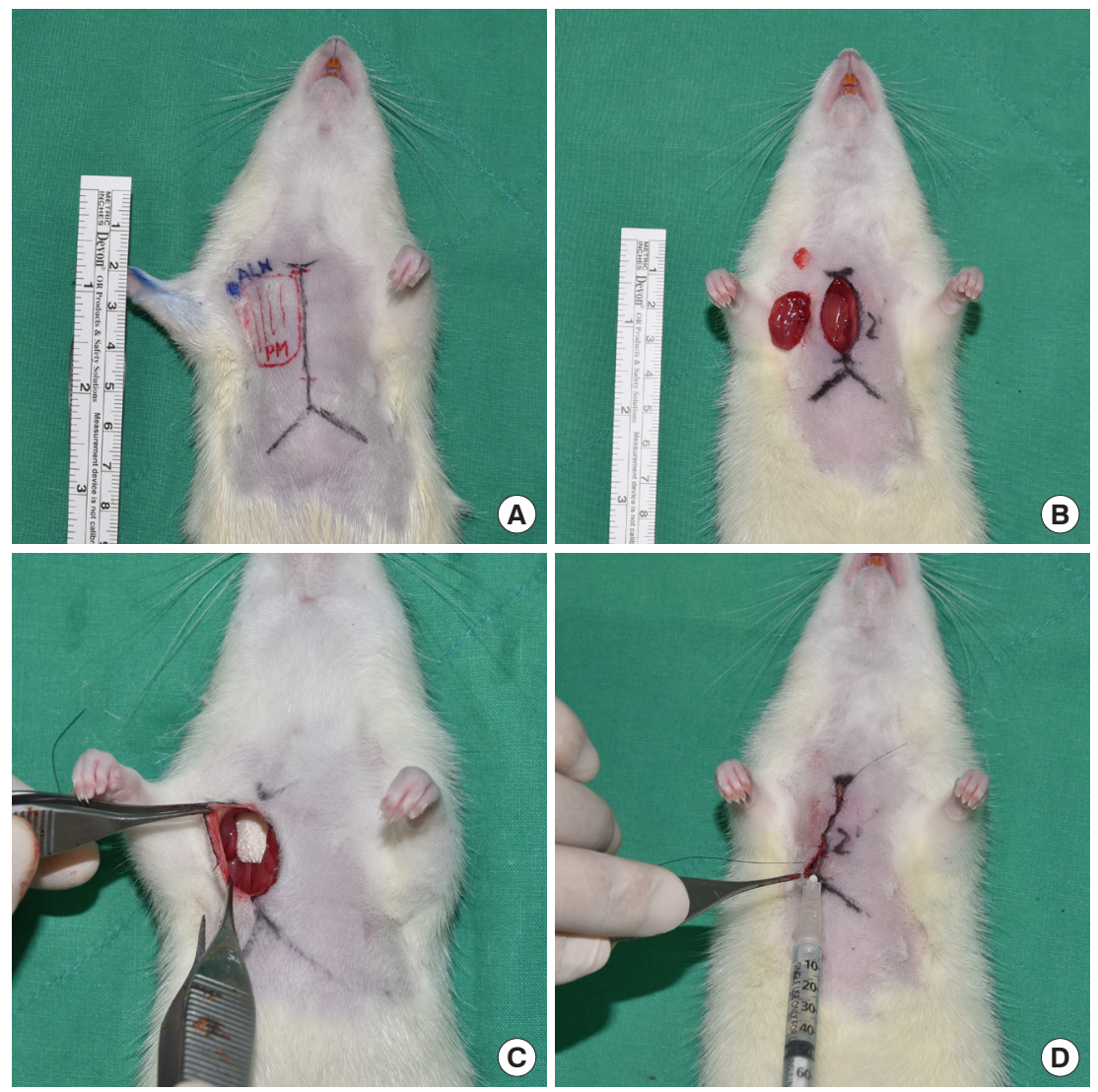

Fig. 1. Surgical procedure in a SpragueDawley rat mastectomy model. (A) Preoperative design. Incision line extending $2 \mathrm{~cm}$ from the jugular notch to the xiphoid. (B) Excised pectoralis major and axillary lymph node. (C) Applying $1 \mathrm{~cm}^{2}$ sized fibrin sealant (Collaseal) to dissected area. (D) After primary closure, appropriate volume of saline was applied to the dissected area. 
dine dressings were performed for 3 days after surgery, with no removal of sutures.

Immediately after suturing, CT images were taken using Quantum FX micro CT ( $90 \mathrm{kVp}$, field of view: $40 \mathrm{~mm}$, with scan times of 2 minutes; PerkinElmer, Hopkinton, MA, USA). At postoperative day (POD) 7, six rats from each group were sacrificed in a $\mathrm{CO}_{2}$ chamber, and CT scans and aspiration were used to measure the amount of seroma. Aspiration was conducted slowly and delicately with 1 -mL syringes and 30-gauge needles, after which the amount was measured in $0.1 \mathrm{~mL}$ units. Subcutaneous flap tissues of the axillary lymph node were fixed in paraffin blocks. The tissues were examined with trichrome and hematoxylin and eosin $(\mathrm{H} \& \mathrm{E})$ staining. At POD 14, seroma volume were measured and histopathological samples taken from the remaining six SD rats in each group. The tissues were observed under an optical microscope to identify hematoma, seroma, congestion, fibroblasts, fibrous and granulation tissue growth, vascular proliferation, necrosis, and inflammation. Cellular and histopathological data were scored semi-quantitatively in scales of 0 to 3 , with $0=$ absent,
$1=$ mild, $2=$ moderate, and $3=$ marked (Figs. 2, 3). All statistical analyses were performed using SPSS software version 22.0 (IBM Corp., Armonk, NY, USA). Differences in seroma volumes among the groups were assessed with one-way analysis of variance for each of the group comparisons. Differences with a value of $\mathrm{P}<0.05$ were considered statistically significant.

\section{Results}

The three groups (group 1: control group, $0.36 \mathrm{~mL}$; group 2: [fibrin sealant, Collaseal] group, $0.424 \mathrm{~mL}$; and group 3: triamcinolone injection group, $0.29 \mathrm{~mL}$ ) had no statistically significant differences in their volume of seroma measured by CT immediately after surgery. In the $\mathrm{CT}$ analysis results at POD 7 of the $18 \mathrm{SD}$ rats taken from the three groups, the seroma levels were significantly lower in group 2 and group 3 compared to group 1 (group 1, $1.01 \mathrm{~mL}$; group 2, $0.27 \mathrm{~mL}$; group 3, 0.26 $\mathrm{mL} ; \mathrm{P}<0.05)$. Aspiration seroma volumes were also significantly lower in group 2 and group 3 than in group 1 (group 1 , $0.95 \mathrm{~mL}$; group 2, $0.2 \mathrm{~mL}$; group 3, $0.28 \mathrm{~mL} ; \mathrm{P}<0.001$ ). At

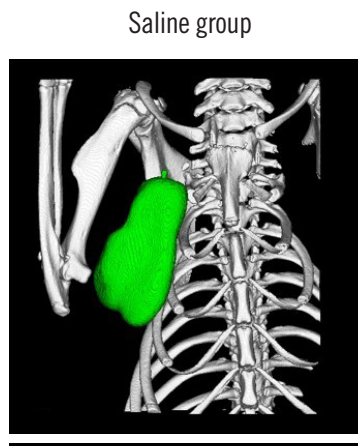

Triamcinolone acetate group
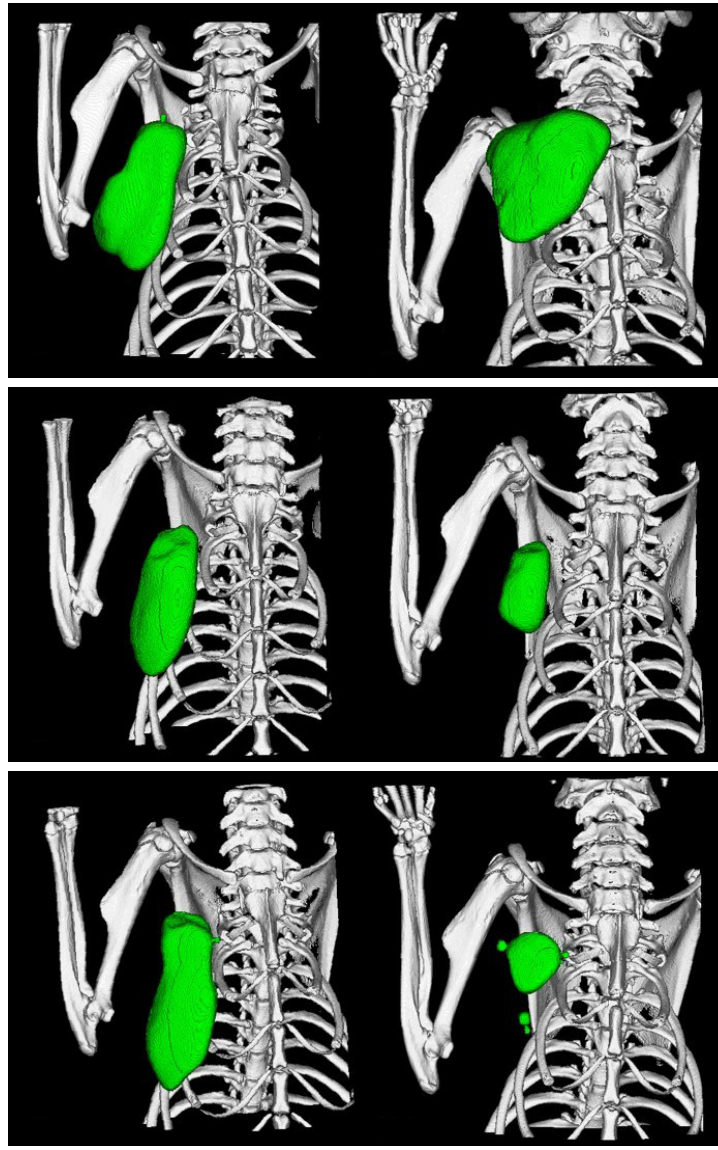

Fibrin sealant group (Collaseal)
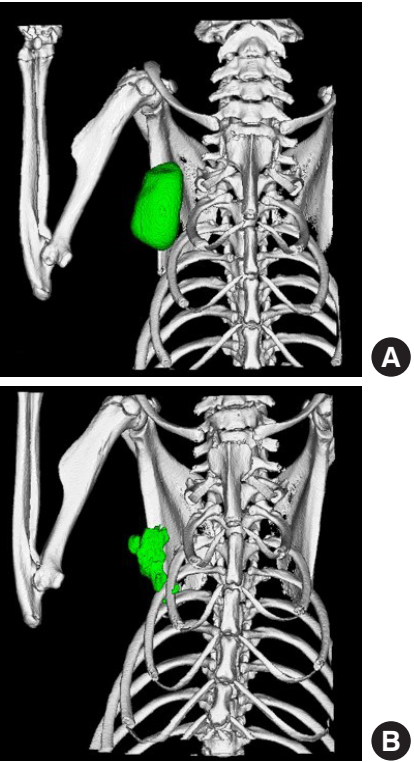

B

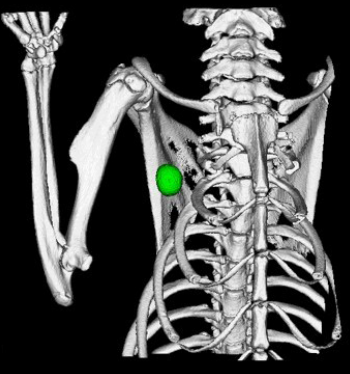

C
Fig. 2. Three-dimensional micro-computed tomography volumetry. (A) Postoperative day (POD) 0, (B) POD 7, and (C) POD 14. 
Kim JS et al.

Fibrin sealant for seroma prevention

JWMR

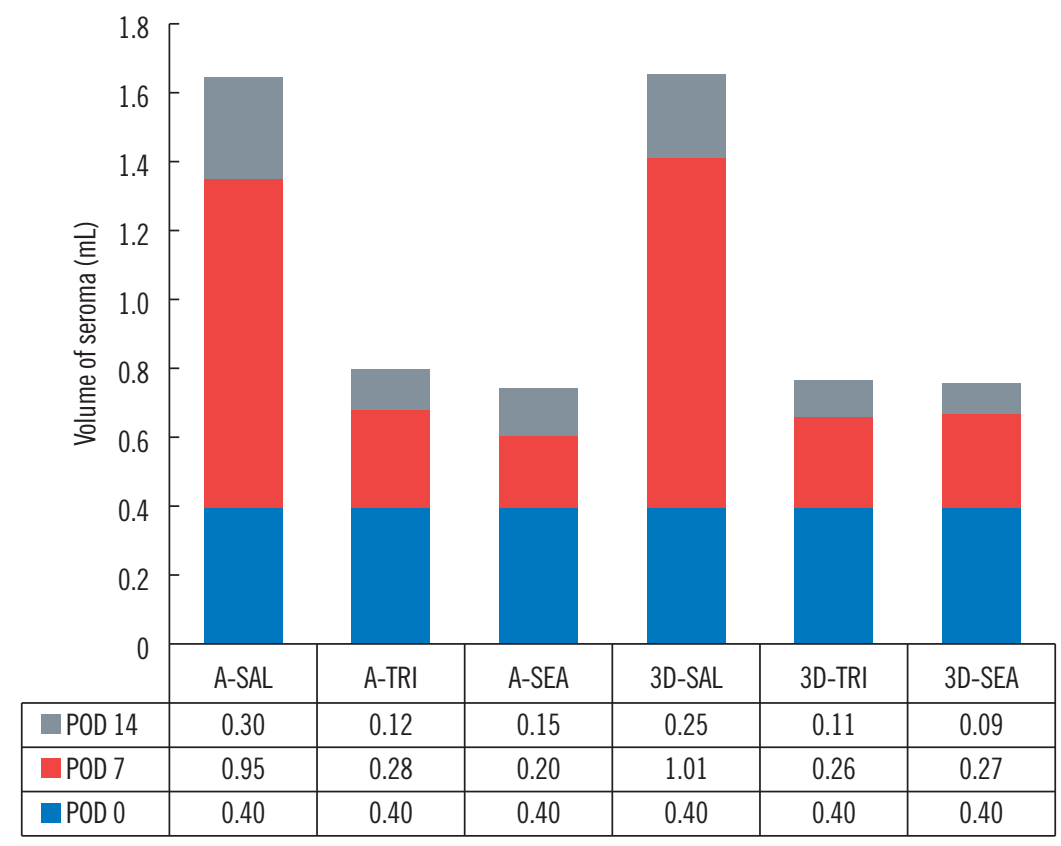

Fig. 3. Volume of seroma. The values measured by the aspiration method and the volumetry of three-dimensional (3D) micro-computed tomography manifest similar amounts of variation. A, aspiration method; SAL, saline group; TRI, triamcinolone acetate group; SEA, fibrin sealant (Collaseal) group; POD, postoperative day.
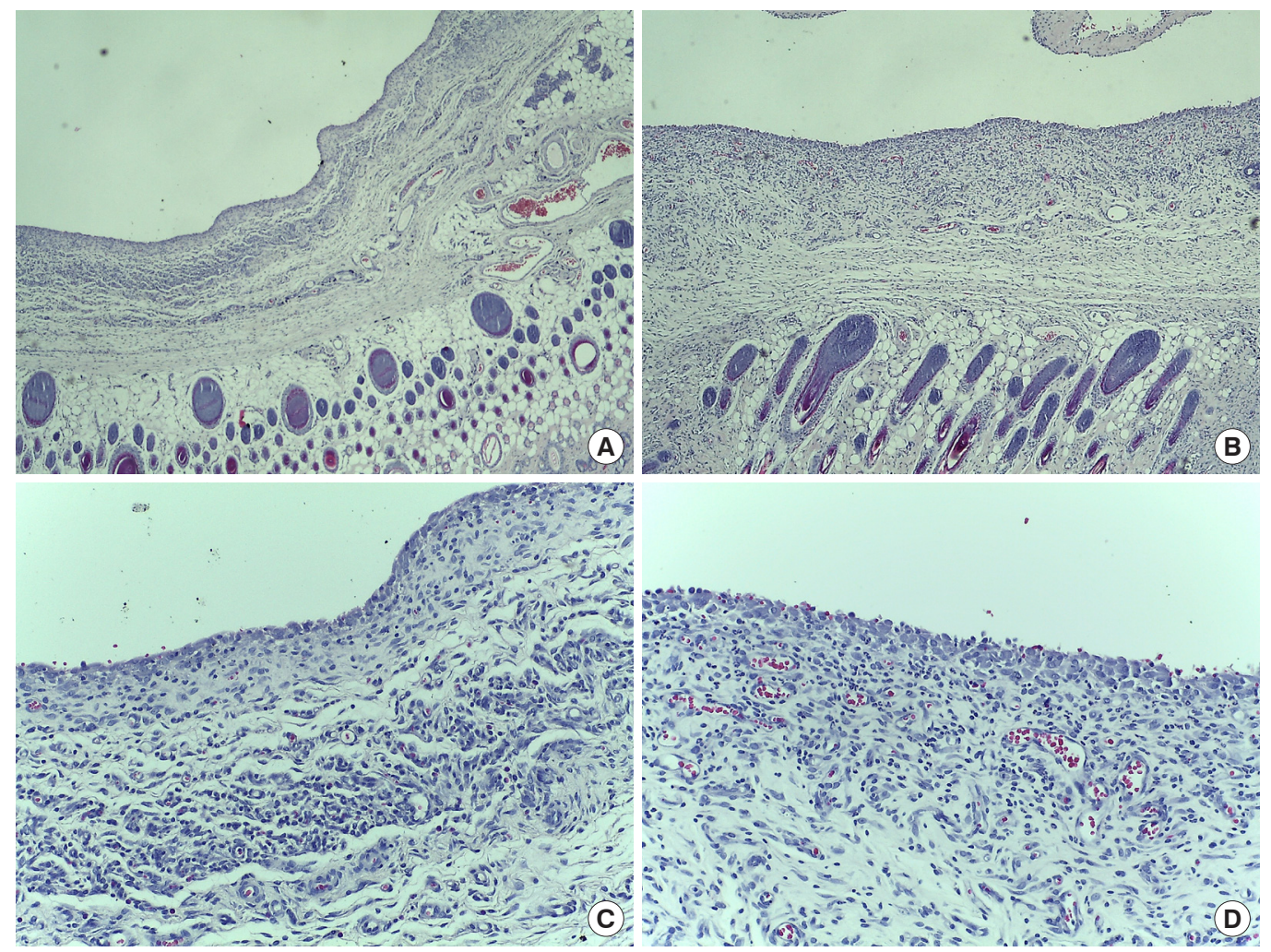

Fig. 4. H\&E stained tissue in the rat mastectomy model. H\&E stain was used to confirm the change of fibroblast layer due to seroma formation in rats. In group 2 (Collaseal), many fibroblasts are observed in a thickened fibroblast layer. (A) Group 1 (normal saline), POD 14 (x40). (B) Group 2 (Collaseal), POD 14 (x40). (C) Group 1, POD 14 (×200). (D) Group 2, POD 14 (x200). H\&E, hematoxylin and eosin; POD, postoperative day. 


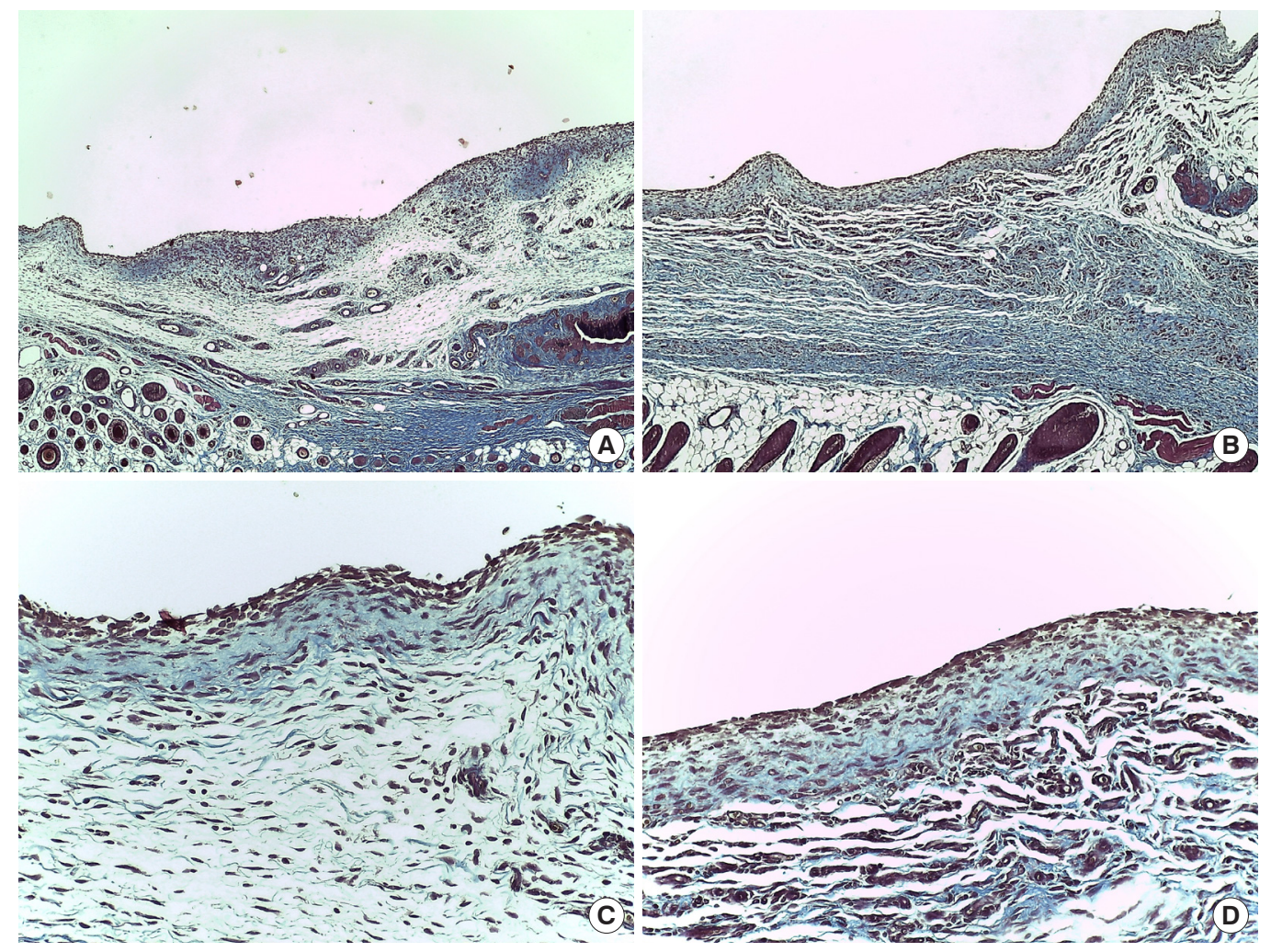

Fig. 5. Trichrome stained tissue in the rat mastectomy model. Trichome stain was used to confirm the change of collagen fiber layer due to seroma formation in rats. In group 2 (Collaseal), abundant collagen fibers are observed and the thickness of the collagen fiber layer is increased. (A) Group 1 (normal saline), POD 14 (×40). (B) Group 2 (Collaseal), POD 14 (×40). (C) Group 1, POD 14 (×200). (D) Group 2, POD 14 (×200). POD, postoperative day.

POD 14, the CT analysis results turned out lower in group 2 and group 3 than in group 1 (group 1, $0.25 \mathrm{~mL}$; group 2, 0.09 $\mathrm{mL}$; group 3, $0.11 \mathrm{~mL} ; \mathrm{P}>0.05$ ), and aspirated seroma volumes were also lower in group 2 and group 3 than in group 1 (group 1, $0.30 \mathrm{~mL}$; group 2, $0.15 \mathrm{~mL}$; group 3, $0.12 \mathrm{~mL} ; \mathrm{P}>0.05$ ). However, on POD 14, there was no statistical significance in either analysis method (Figs. 2, 3). All rats survived without major complications (e.g., disruption, flap necrosis, infection) until the end of the experiment. Two rats injected with triamcinolone sustained mild erythema and swelling for several days due to inflammation and then improved.

Histopathologic examination demonstrated that both angiogenesis and wound healing were more active in group 2 compared to groups 1 and 3. In trichrome staining, inflammation (granulocytes and giant cells) was observed more frequently in group 1, and the inflammation also lasted longer. This confirms that inflammation reaction was reduced in group 2 and group 3, helping to prevent the formation of seroma (Figs. 4, 5). In addition, in the process of collecting the histologic sections, it was confirmed that Collaseal was absorbed gradually in group 2 , and the absorption was completed by POD 14.

\section{Discussion}

Several papers have recommended a variety of methods to reduce the formation of seroma. These methods usually include reducing dead space during surgery, and other methods include the use of sclerosing agents (fibrin glue, tetracycline, hydrochloric acid), anti-inflammatory drugs, and chemotherapeutic agents to reduce or prevent postoperative drainage.

Collaseal is a wound dressing foam product for secondary intention healing approved by the Ministry of Food and Drug Safety and is a bi-membrane product made of a porous membrane and an adhesive membrane. The white porous membrane is in the form of a sponge and contains type 1 collagen and hyaluronic acid, absorbing exudates and helping heal wounds. The yellow adhesive membrane is a film containing type 1 collagen, thrombin, and levodopa, which enhances adhesion to tissues and helps the hemostasis of wound areas 
$[12,13]$. Collagen, a major component, is the most abundant structural protein that accounts for $20 \%$ to $30 \%$ of human protein and is a fibrous protein that constitutes the skin and connective tissue of mammals. Collagen improves wound healing in the manner described above. Levodopa, which is added in a small amount to the adhesive membrane, is an adhesive amino acid that enhances adhesiveness with tissues. Thrombin induces aggregation of fibrinogen at the bleeding site to help hemostasis $[14,15]$.

For this reason, we hypothesized that using Collaseal would help prevent and reduce the seroma that occurs after reconstructive breast surgery. In our rat mastectomy model, triamcinolone acetate was used on a control group, which had been proven to be effective in reducing seroma $[2,4]$. There is the secondary advantage of the sealing effect when applying patch-type Collaseal directly where leakage is suspected. Most previous studies reported outcomes using a variety of liquidtype agents; hardly any studies used patch-type materials like Collaseal.

Our seroma animal model used the one-sided (right) mastectomy and axillary lymph node dissection as described by Harada et al. [9], and a classical needle aspiration method to measure the amount of seroma after surgery. 3D micro-CT volumetry analysis was also performed to further measure the amount of seroma precisely. This is the currently recommended method for measuring the amount of change in seroma, as it is convenient and noninvasive [16,17].

3D micro-CT volumetry analysis and aspiration results on POD 7 found significantly decreased seroma in groups 2 and 3 compared to group 1. This proves the roles of Collaseal and triamcinolone injection in reducing seroma formation.

During the experiment, although there was mild linear necrosis on POD 7 in one individual from group 1 and one from group 3, applying ointment improved the necrosis without disruption, and the necrosis was no longer visible on POD 14. Though hematoma was not observed on POD 7, it was observed in two individuals in group 1 and in one in group 3 on POD 14. No wound infections or premature deaths were observed.

In histopathological examinations, $\mathrm{H} \& \mathrm{E}$ staining is the best method to determine normal and pathologic structures. As it makes the structure immediately visible, it is also the easiest and most economical method to confirm angiogenesis. The staining indicated good angiogenesis in group 2, demonstrating that these materials could be helpful in wound healing. However, one of this study's limitations is that angiogenesis was not further confirmed with VEGF or CD31/PECAM-1 staining.

Meanwhile, Trichrome staining also confirmed that inflammation was reduced in the Collaseal group. Reducing the duration of inflammation accelerates wound healing. This, together with enhanced angiogenesis, presumably acts toward the seroma-reducing effect of the collagen-enhanced fibrin sealant (Collaseal) after mastectomy in SD rats.

In conclusion, we report that collagen-enhanced fibrin sealant (Collaseal) and triamcinolone injection in a rat mastectomy seroma model resulted in a decrease in seroma compared to the group injected with saline. The collagen-enhanced fibrin sealant (Collaseal) has been shown to increase adhesion, neovascularization, growth of granulation tissue and to accelerate wound healing by supplying the necessary ingredients. Therefore, a reliable outcome can be expected by alternating other treatments with use of this patch-type material that promotes wound healing and prevents seroma formation.

\section{Conflict of interest}

No potential conflict of interest relevant to this article was reported.

\section{ORCID iDs}

Jong Seong Kim https://orcid.org/0000-0002-4725-9277

Pil Seon Eo https://orcid.org/0000-0003-1909-7298

Joon Seok Lee https://orcid.org/0000-0002-1580-0487

Jeong Woo Lee https://orcid.org/0000-0003-4903-6066

Kang Young Choi https://orcid.org/0000-0002-7735-1939

Ho Yun Chung https://orcid.org/0000-0001-7359-3044

Byung Chae Cho https://orcid.org/0000-0002-9395-8896

Jeeyeon Lee

Ho Yong Park

Jung Dug Yang

https://orcid.org/0000-0003-1826-1690

https://orcid.org/0000-0002-4380-0089

https://orcid.org/0000-0002-9040-4724

\section{References}

1. Yan WH, Mang JB, Ren LL, et al. Natural history of seroma following the immediate latissimus dorsi flap method of breast reconstruction. Chin Med J (Engl) 2018;131:1674-9.

2. Hart AM, Duggal C, Pinell-White X, et al. A prospective randomized trial of the efficacy of fibrin glue, triamcinolone acetonide, and quilting sutures in seroma prevention after latissimus dorsi breast reconstruction. Plast Reconstr 
Surg 2017;139:854e-863e.

3. Lee J, Bae Y, Jung JH, et al. Effects of quilting suture interval on donor site seromas after breast reconstruction with latissimus dorsi muscle flap: a randomized trial. Clin Breast Cancer 2016;16:e159-64.

4. Taghizadeh R, Shoaib T, Hart AM, et al. Triamcinolone reduces seroma re-accumulation in the extended latissimus dorsi donor site. J Plast Reconstr Aesthet Surg 2008;61:63642.

5. Kim JS, Eo PS, Lee JS, et al. Use of Abnobaviscum to treat refractory seroma after breast reconstruction with a latissimus dorsi flap: a case report. Archives of Aesthetic Plastic Surgery 2019;25:73-8.

6. Di Monta G, Caraco C, Crispo A, et al. Collagen sealant patch to reduce lymphatic drainage after lymph node dissection. World J Surg Oncol 2012;10:275.

7. Burak WE Jr, Goodman PS, Young DC, et al. Seroma formation following axillary dissection for breast cancer: risk factors and lack of influence of bovine thrombin. J Surg Oncol 1997;64:27-31.

8. Stehbens WE. Postmastectomy serous drainage and seroma: probable pathogenesis and prevention. ANZ J Surg 2003;73:877-80.

9. Harada RN, Pressler VM, McNamara JJ. Fibrin glue reduces seroma formation in the rat after mastectomy. Surg Gynecol Obstet 1992;175:450-4.

10. Aitken DR, Hunsaker R, James AG. Prevention of seromas following mastectomy and axillary dissection. Surg Gynecol Obstet 1984;158:327-30.

11. Turel KS, Dilek ON, Akbulut G, et al. Effects of local antiinflammatory drugs on seroma formation after mastectomy and axillary lymph node dissection in the rat model. Natl J Med Res 2014;4:228-31.

12. Berger A, Tempfer C, Hartmann B, et al. Sealing of postoperative axillary leakage after axillary lymphadenectomy using a fibrin glue coated collagen patch: a prospective randomised study. Breast Cancer Res Treat 2001;67:9-14.

13. Uden P, Aspegren K, Balldin G, et al. Fibrin adhesive in radical mastectomy. Eur J Surg 1993;159:263-5.

14. Sanders RP, Goodman NC, Amiss LR Jr, et al. Effect of fibrinogen and thrombin concentrations on mastectomy seroma prevention. J Surg Res 1996;61:65-70.

15. Wang JY, Goodman NC, Amiss LR Jr, et al. Seroma prevention in a rat mastectomy model: use of a light-activated fibrin sealant. Ann Plast Surg 1996;37:400-5.

16. Kader HA, Truong PT, Pai R, et al. When is CT-based postoperative seroma most useful to plan partial breast radiotherapy? Evaluation of clinical factors affecting seroma volume and clarity. Int J Radiat Oncol Biol Phys 2008;72: 1064-9.

17. Yang TJ, Minkema D, Elkhuizen PH, et al. Clinical applicability of cone-beam computed tomography in monitoring seroma volume change during breast irradiation. Int J Radiat Oncol Biol Phys 2010;78:119-26. 